\title{
Broken one-particle symmetry in few-electron coupled quantum dots
}

\author{
T. Chwiej ${ }^{1}$ S. Bednarek, ${ }^{1}$ B. Szafran, ${ }^{1,2}$ J. Adamowski, ${ }^{1, *}$ and F. M. Peeters ${ }^{2, \dagger}$ \\ ${ }^{1}$ Faculty of Physics and Applied Computer Science, AGH University of Science and Technology, PL-30059 Kraków, Poland \\ ${ }^{2}$ Departement Fysica, Universiteit Antwerpen (UA/CGB), Groenenborgerlaan 171, B-2020 Antwerpen, Belgium
}

(Received 29 September 2005; published 15 February 2006)

\begin{abstract}
Few-electron systems confined in vertically coupled quantum dots are studied by exact methods and through the local spin density approximation (LSDA). Special attention is paid to the recovering of the one-particle symmetry properties in the LSDA. It is shown that-in spite of accurate energy estimates - the LSDA does not reproduce the one-particle parity and the relative probability of finding a definite number of electrons in the different quantum dots. This symmetry breaking appears for certain electron configurations and intermediate thickness of the interdot barrier and is a direct result of electron-electron correlation. We discuss the effect of correlation on the symmetry properties of the few-electron wave function and determine the limits of the applicability of the LSDA for coupled quantum dots.
\end{abstract}

DOI: 10.1103/PhysRevB.73.075422

PACS number(s): 73.21.La, 73.22.Gk

\section{INTRODUCTION}

Quantum states of few-electron systems confined in coupled quantum dots (QDs) ${ }^{1-9}$ are usually studied by meanfield methods, e.g., Hartree-Fock (HF) ${ }^{10-12}$ method and local spin density approximation (LSDA), ${ }^{13-17}$ which are based on the one-electron approximation. In the simplest version of these methods, electron-electron correlation is either neglected (in the restricted HF approach ${ }^{11,12,18}$ ) or treated in an oversimplified manner (in the LSDA ${ }^{13-17}$ ). Electron-electron correlation is much better taken into account in the unrestricted version of the HF method ${ }^{11,12,18}$ and in the configuration interaction (CI) method, ${ }^{19,20}$ which leads to an improvement of the energy estimates. In the present paper, the influence of electron-electron correlation on the accuracy of the LSDA solution is studied. When testing the accuracy of the LSDA method, we should determine not only the energy of the system, but also the symmetry properties of the manyelectron wave function. In particular, due to the one-electron character of the LSDA, it is important to check if the oneelectron LSDA wave functions reproduce the symmetry of the exact many-electron solutions.

The broken-symmetry LSDA solutions were analyzed by Harju et al. ${ }^{17}$ for a four-electron rectangular QD. Pi et al. ${ }^{14}$ studied the addition spectra of vertically coupled QDs and found that introducing asymmetry between the QDs into the LSDA leads to a better agreement with experiment. A systematic study of the occupancy of bonding and antibonding orbitals in two vertically coupled QDs with three up to six electrons in the presence of a magnetic field was made by Partoens and Peeters. ${ }^{21}$ Rontani et $a l .{ }^{19}$ applied the CI method to study the magnetic-field influence on the occupancy of electronic bonds for vertically coupled QDs with $N=2, \ldots, 7$ electrons. In a recent paper, Austing et al. ${ }^{22}$ reported different electron phases at integer filling factor in vertically coupled QDs and analyzed the experimental results within the LSDA.

The properties of electrons confined in coupled QDs depend on the parameters of the potential barrier layer separating the QDs. ${ }^{21}$ In particular, the coupling between the QDs can be tuned by changing the height and thickness of the barrier layer. If the barrier layer is sufficiently thin to enable tunneling of electrons between the QDs, then the nanostructure exhibits properties similar to that of a single QD. If however the barrier layer is thick enough to prevent tunneling, the electrons become localized in the different QDs. Then, we deal with two isolated electrostatically coupled QDs. ${ }^{23}$

When using the LSDA we reduce the many-electron problem to the one-electron problem, in which the electronelectron interactions are described by an effective potential obtained through a self-consistent procedure. In the LSDA we can employ one-electron wave functions, on which we impose the same symmetry as that of the confining potential. In analogy to the restricted HF method, this version of the LSDA will be called the restricted LSDA ( $r$-LSDA) throughout the present paper. However, the symmetry requirement of the $r$-LSDA is too restrictive, since only the total manyelectron wave function should possess the symmetry of the external confinement potential. The one-electron wave functions do not have to possess this symmetry and therefore the effective potential can exhibit a different symmetry from that of the confinement potential. The $r$-LSDA method provides fairly accurate energy estimates for few-electron systems. However, for certain electron configurations the $r$-LSDA leads to unreliable results and the predicted electron groundstate configuration turned out to be incorrect. ${ }^{16}$

The above-mentioned shortcomings of the restricted LSDA are removed in the unrestricted version of the LSDA ( $u$-LSDA), in which the one-electron wave functions can possess a lower symmetry ${ }^{17,25,26}$ than the confinement potential. In the $u$-LSDA, we are dealing with the breaking of the one-particle symmetry, which leads to more accurate results for the energy. ${ }^{17}$

The purpose of the present paper is to study the symmetry properties of the few-electron system confined in vertically coupled QDs (artificial molecules) and to test the applicability of the $r$-LSDA and $u$-LSDA. Detailed tests have been performed for the two-, three-, and four-electron systems, for which we were able to obtain exact results and compare them with those of the LSDA. For the two-electron coupled QDs, we obtain exact results by separating the center-of-mass mo- 
tion and solving the relative-motion problem by accurate finite-difference numerical method. ${ }^{24}$ For the three- and fourelectron coupled QDs, the exact results are obtained with the help of the CI method. Particular attention is paid to the conditions, under which the LSDA one-electron wave functions reproduce the proper symmetry of the system. Although the LSDA wave functions do not always exhibit the correct symmetry, they usually lead to accurate energy estimates. Therefore, we have compared the electron addition energies as obtained from the $r$-LSDA and $u$-LSDA for the $N$-electron artificial molecule with $2 \leqslant N \leqslant 13$.

The paper is organized as follows: the theoretical methods applied are described in Sec. II, the results are presented in Sec. III, and conclusions and summary are presented in Sec. IV. In the Appendix we provide formulas for the effective electron-electron interaction potentials as derived for different quantum states.

\section{THEORY}

We study the systems of $N=2,3$, and 4 electrons confined in a vertical double coupled QD nanostructure. ${ }^{1,2}$ The confinement potential $V_{\|}(z)$ in the vertical direction is taken as two identical potential wells with thickness $Z=12 \mathrm{~nm}$ and depth $V_{0}=200 \mathrm{meV}$, separated by a barrier with varying thickness $b$. In the lateral directions, we assume parabolic confinement

$$
V_{\perp}(x, y)=\frac{m_{e} \omega_{0}^{2}}{2}\left(x^{2}+y^{2}\right),
$$

with the confinement energy $\hbar \omega_{0}=10 \mathrm{meV}$. For our model nanostructure we employ the parameters of GaAs, i.e., electron effective mass $m_{e}=0.067 m_{e 0}$, where $m_{e 0}$ is the free electron mass, and dielectric constant $\varepsilon=12.4$.

\section{A. Center-of-mass separation for two-electron system}

For two electrons confined in the lateral parabolic potential we can separate ${ }^{24,27}$ the center-of-mass (CM) motion and relative motion, which leads to the center-of-mass Hamiltonian

$$
H_{\mathrm{CM}}=-\frac{\hbar^{2}}{4 m_{e}} \nabla_{\mathrm{CM}}^{2}+m_{e} \omega_{0}^{2} \varrho_{\mathrm{CM}}^{2}
$$

and the relative Hamiltonian

$$
\begin{aligned}
H_{\text {rel }}= & -\frac{\hbar^{2}}{m_{e}} \nabla_{12}^{2}+\frac{m_{e} \omega_{0}^{2}}{4} \varrho_{12}^{2}-\frac{\hbar^{2}}{2 m_{e}}\left(\frac{\partial^{2}}{\partial z_{1}^{2}}+\frac{\partial^{2}}{\partial z_{2}^{2}}\right) \\
& +V_{\|}\left(z_{1}\right)+V_{\|}\left(z_{2}\right)+\frac{\kappa e^{2}}{\varepsilon r_{12}},
\end{aligned}
$$

where $\vec{\varrho}_{\mathrm{CM}}=\frac{1}{2}\left(x_{1}+x_{2}, y_{1}+y_{2}\right), \vec{\varrho}_{12}=\left(x_{1}-x_{2}, y_{1}-y_{2}\right), \vec{\nabla}_{\mathrm{CM}}$ and $\vec{\nabla}_{12}$ are the nabla operators, which contain the derivatives with respect to the components of vectors $\vec{\varrho}_{\mathrm{CM}}$ and $\vec{\varrho}_{12}$, respectively, $r_{12}=\left[\left(x_{1}-x_{2}\right)^{2}+\left(y_{1}-y_{2}\right)^{2}+\left(z_{1}-z_{2}\right)^{2}\right]^{1 / 2}$, and $\kappa$ $=1 /\left(4 \pi \varepsilon_{0}\right)$. The solutions of the eigenproblem for the Hamiltonian $H_{\mathrm{CM}}$ are the wave functions of the two- dimensional harmonic oscillator associated with eigenvalues $E_{N_{\mathrm{CM}} M_{\mathrm{CM}}}=\left(2 N_{\mathrm{CM}}+\left|M_{\mathrm{CM}}\right|+1\right) \hbar \omega_{0}$, where $N_{\mathrm{CM}}$ and $M_{\mathrm{CM}}$ are the radial and $z$-component angular momentum quantum numbers, respectively. The eigenproblem of the relativemotion Hamiltonian $H_{\text {rel }}$ cannot be solved analytically, therefore, we must rely on a numerical solution. For this purpose, we transform the relative-motion eigenequation to cylindrical coordinates, separate out the angular dependence, and ascribe a definite $z$ angular-momentum component to each eigenstate, which reduces the number of independent coordinates to three. For this reduced eigenproblem we apply the imaginary-time step method ${ }^{28}$ on a three-dimensional mesh. This method enables us to obtain numerical solutions with any required accuracy. Throughout the present paper we will treat these numerical solutions as exact.

\section{B. Configuration interaction method for three- and four- electron systems}

For three and four electrons the center-of-mass separation leads to a relative-motion problem with too many independent variables, which does not allow for an efficient numerical solution. Therefore, in order to obtain the exact solutions for the three- and four-electron artificial molecules, we apply the CI method. We employ the CI basis of Slater determinants constructed from different configurations of the oneelectron wave functions, which take into account the correlation effects in the vertical direction.

Below we briefly describe the $\mathrm{CI}$ approach used in the present paper. The $N$-electron Hamiltonian has the form

$$
H=\sum_{i=1}^{N} h_{i}+\sum_{i=1}^{N} \sum_{j>i}^{N} \frac{\kappa e^{2}}{\varepsilon\left|\vec{r}_{i}-\vec{r}_{j}\right|},
$$

where the one-electron Hamiltonian

$$
h_{i}=h_{\|}+h_{\perp}
$$

is a sum of the vertical-motion Hamiltonian

$$
h_{\|}=-\frac{\hbar^{2}}{2 m_{e}} \frac{\partial^{2}}{\partial z^{2}}+V_{\|}(z)
$$

and the lateral-motion Hamiltonian

$$
h_{\perp}=-\frac{\hbar^{2}}{2 m_{e}}\left(\frac{\partial^{2}}{\partial x^{2}}+\frac{\partial^{2}}{\partial y^{2}}\right)+\frac{m_{e} \omega_{0}^{2}}{2}\left(x^{2}+y^{2}\right) .
$$

The eigenfunctions of the one-electron Hamiltonian (5) can be expressed as

$$
\psi_{\mu \nu}(x, y, z)=f_{\mu}(z) g_{\nu}(x, y),
$$

where $f_{\mu}(z)$ and $g_{\nu}(x, y)$ describe the vertical and lateral motion, respectively. Wave functions $f_{\mu}(z)$ are obtained from a numerical diagonalization of the finite-difference version of Hamiltonian (6) on a one-dimensional mesh. In the present approach, we included the four lowest-energy states $f_{\mu}(z)$. Due to the simple rectangular shape of the vertical confinement potential, taking into account a larger number of states $f_{\mu}(z)$ does not change the results. In this way, we can fully describe the electron-electron correlation in the vertical direction. 
For the wave functions $g_{\nu}(x, y)$ we take the eigenfunctions of the two-dimensional harmonic oscillator with frequency $\omega$ (not necessarily equal to $\omega_{0}$ ), corresponding to the angular momenta 0 and $\pm \hbar$. Their explicit forms are

$$
g_{0}(x, y)=\sqrt{\frac{2 \beta}{\pi}} \exp \left[-\beta\left(x^{2}+y^{2}\right)\right]
$$

and

$$
g_{ \pm 1}(x, y)=\frac{2 \beta}{\sqrt{\pi}}(x \pm i y) \exp \left[-\beta\left(x^{2}+y^{2}\right)\right],
$$

where $\beta=m_{e} \omega / 2$. We checked that including the larger number of wave functions $g_{\nu}(x, y)$ leads to a slight lowering of the energy, which however does not change the parity of the lowest-energy states.

Wave functions $f_{\mu}(z)$ and $g_{\nu}(x, y)$ together with two eigenstates of the $z$ spin component form the basis consisting of 24 one-electron orthogonal spin orbitals, from which we can construct $\mathcal{N}_{S}=24 ! /[(24-N !) N$ !] Slater determinants. The $N$-electron states are also the eigenstates of the following operators: $z$ component of the total orbital momentum $\left(\hat{L}_{z}\right)$, square of the total spin $\left(\hat{S}^{2}\right), z$ component of the total spin $\left(\hat{S}_{z}\right)$, and total parity $(\hat{\Pi})$. Among the $\mathcal{N}_{S}$ Slater determinants, we choose only those which possess the required parity and are eigenfunctions of $\hat{S}_{z}$. The actual maximum number of basis elements used in our calculations was 1343 for $N=4$. We identify the states with the different eigenvalues of $\hat{S}^{2}$ by determining the degree of their degeneracy with respect to eigenvalues of $\hat{S}_{z}$. In the present paper, $\omega$ is treated as a variational parameter; so, in general, $\omega \neq \omega_{0}$. When performing a minimization over the variational parameter $\omega$, we obtain $\omega<\omega_{0}$, which lowers the energy of interaction between the electrons confined in the same QD. The optimum values of $\hbar \omega$ increase with barrier thickness $b$ increasing from 0 to $10 \mathrm{~nm}$ and vary from $6.5 \mathrm{meV}$ to $7.5 \mathrm{meV}$ for the four-electron ground state and from $7.5 \mathrm{meV}$ to $8.5 \mathrm{meV}$ for the three-electron ground state. In the Appendix we provide the interelectron interaction matrix elements calculated with the use of our basis functions $g_{\nu}(x, y)$.

\section{Local spin density approximation}

The LSDA is based on the Kohn-Sham equation

$$
\left(-\frac{\hbar^{2}}{2 m_{e}} \nabla^{2}+V_{\mathrm{ext}}+V_{\mathrm{eff}}^{\alpha \sigma}\right) \psi_{\alpha \sigma}=\varepsilon_{\alpha \sigma} \psi_{\alpha \sigma},
$$

which describes the electron in the orbital state $\alpha$ with $z$ spin component $\sigma$. In Eq. (11), $V_{\text {ext }}$ is the external (confinement) potential and $V_{\text {eff }}^{\alpha \sigma}$ is the effective potential, which takes into account the interactions between the electrons. We assume that the spin of each electron is well defined and the spindependent part of the wave function can be separated out from the orbital part. Moreover, due to the cylindrical symmetry of $V_{\text {ext }}$ and $V_{\text {eff }}^{\alpha \sigma}$, the one-electron solutions of Eq. (11) have a well-defined $z$ component of angular momentum. They can be written down in cylindrical coordinates as follows:

$$
\psi_{n m \sigma}(r, z, \phi)=\varphi_{n m}(r, z) e^{i m \phi} \chi_{\sigma},
$$

where $m=0, \pm 1, \ldots$ is the quantum number of the $z$ component of orbital momentum and $n=1,2, \ldots$ numbers the subsequent solutions with definite $m$ and $\sigma$. The eigenvalues of the $z$ components of the total angular momentum and total spin are equal to (in units $\hbar$ ): $M_{z}=\sum_{i=1}^{N} m_{i}$ and $S_{z}=\sum_{i=1}^{N} \sigma_{i}$, respectively. Throughout the present paper only the states with $M_{z} \geqslant 0$ and $S_{z} \geqslant 0$ are considered, since the external magnetic field is absent and the spin-orbit interaction has been neglected.

We solve the Kohn-Sham equation (11) using the imaginary-time step method ${ }^{28}$ on two-dimensional mesh $\left(r_{i}, z_{j}\right)$. The simultaneous application of the Schmidt orthogonalization enables us to obtain the excited one-electron states. We denote the $N$-electron states, as calculated by the LSDA, by specifying all the occupied one-electron orbitals $(\mathrm{nm})$. The states with $|m|=0,1,2, \ldots$ are labelled by $s, p$, $d, \ldots$, respectively. For example, the two-electron singlet state with $M_{z}=0$ is denoted by $1 s^{2}$.

The effective potential in Eq. (11) is the sum of the Hartree and exchange-correlation potentials, i.e.,

$$
V_{\mathrm{eff}}^{\alpha \sigma}(\vec{r})=V_{H}^{\alpha \sigma}(\vec{r})+V_{x c}^{\alpha \sigma}(\vec{r}) .
$$

We calculate both the components of the effective potential taking into account the self-interaction corrections according to the method of Perdew and Zunger. ${ }^{29}$ The exchangecorrelation potential is a difference of two functionals one of which depends on electron densities $\varrho^{\uparrow}$ and $\varrho^{\downarrow}$ while the second depends on $\varrho^{\alpha \beta}$ only. The electron density $\varrho^{\sigma}$, which corresponds to the well-defined spin $\sigma=\uparrow, \downarrow$, is calculated as the sum $\varrho^{\sigma}(\vec{r})=\Sigma_{\beta}\left|\psi_{\beta \sigma}(\vec{r})\right|^{2}$ over all occupied states with spin $\sigma$. The electron density in the considered state is given by $\varrho^{\alpha \sigma}(\vec{r})=\left|\psi_{\alpha \sigma}(\vec{r})\right|^{2}$. The exchange-correlation potential $V_{x c}\left(\left[\varrho^{\uparrow}, \varrho^{\downarrow}\right], \vec{r}\right)$ is taken on in the parametrization of Ceperley and Alder. ${ }^{30}$ In a similar way, we calculate the Hartree potential for each state separately by extracting from the total electron density the electron density of the state under consideration. Accordingly, the Hartree potential is determined as follows:

$$
V_{H}^{\alpha \sigma}(\vec{r})=\frac{\kappa e^{2}}{\varepsilon} \int d^{3} r^{\prime} \frac{\varrho\left(\vec{r}^{\prime}\right)}{\left|\vec{r}-\vec{r}^{\prime}\right|},
$$

where $\varrho(\vec{r})=\varrho^{\uparrow}(\vec{r})+\varrho^{\downarrow}(\vec{r})-\varrho^{\alpha \sigma}(\vec{r})$. The exchange-correlation potential is calculated on the same mesh, on which we solve Eq. (11). In the framework of the LSDA method, we employ the electron densities determined on the same grid points. In order to calculate the Hartree potential using Eq. (14), for each grid point one must perform the summation over all the grid points. This means that the computational complexity is a quadratic function of the number of grid points, i.e., for the large mesh the computation is very time consuming. In order to accelerate the computations, we use Eq. (14) to calculate the Hartree potential only on the cylindrical surface, which encompasses the computational region. This leads to the boundary condition on the Hartree potential, which is calculated inside the cylinder by the Poisson equation. 
In the present paper, we have applied the following two versions of the LSDA: restricted LSDA ( $r$-LSDA) and unrestricted LSDA ( $u$-LSDA). In the $r$-LSDA, the one-electron wave functions reproduce the symmetry of the external confinement potential, while in the $u$-LSDA this symmetry can be broken if the broken-symmetry solution results in the lower energy.

\section{One-electron parity and relative probability}

The external confinement potential is symmetric against inversion, i.e., the transformation $(x, y, z) \rightarrow(-x,-y,-z)$ and therefore the parity operator commutes with the Hamiltonian of the $N$-electron system. This implies that the total $N$-electron wave function must possess a well-defined parity. However, it is not necessary that the symmetry of the manyelectron wave function is mapped onto the symmetry of the one-electron wave functions used in the LSDA. As a consequence, it is possible that the effective electron-electron interaction potential, used in the one-electron Kohn-Sham equation [Eq. (11)], does not exhibit parity symmetry and consequently the eigenfunctions of Eq. (11) do not necessarily possess a well-defined parity.

The breaking of the symmetry of the one-electron wave functions is a consequence of the inclusion of electronelectron correlation and can lead to a lowering of the total ground-state energy when calculated within the LSDA. ${ }^{13}$ In the LSDA, the approximate many-electron wave function is constructed from the one-electron wave functions, which determine its symmetry. In the present paper, we focus on the correlation and symmetry properties of the few-electron system in the vertical $(z)$ direction. Therefore, in order to diminish the correlation effects in the lateral directions, we assume a strong lateral confinement potential with confinement energy $\hbar \omega_{0}=10 \mathrm{meV}$. Owing to the strong lateral confinement, the Coulomb interaction only slightly modifies the lateral shape of the one-electron wave functions.

In order to determine quantitatively the effect of correlation on the one-electron states, we introduce two auxiliary quantities, namely, the one-electron $z$ parity with operator $\hat{\pi}$ and relative probability $P\left(N_{1} \mid N_{2}\right)$ of finding $N_{1}$ electrons in one QD and $N_{2}$ electrons in the other. The estimates of both these quantities obtained with the LSDA methods will be compared with the exact results. Such comparison enables us to check, to which extent the LSDA wave functions reproduce the properties of the exact wave function.

The operator $\hat{\pi}$ of the one-electron $z$ parity is defined as follows: when operating with $\hat{\pi}$ on the total wave function $\Psi_{N}\left(x_{1}, y_{1}, z_{1}, \sigma_{1}, \ldots, x_{N}, y_{N}, z_{N}, \sigma_{N}\right)$ of $N$ indistinguishable electrons the sign of the $z$ coordinate of one electron is changed. Explicitly,

$$
\begin{aligned}
& \hat{\pi} \Psi_{N}\left(x_{1}, y_{1}, z_{1}, \sigma_{1}, \ldots, x_{N}, y_{N}, z_{N}, \sigma_{N}\right) \\
& \quad=\Psi_{N}\left(x_{1}, y_{1},-z_{1}, \sigma_{1}, \ldots, x_{N}, y_{N}, z_{N}, \sigma_{N}\right),
\end{aligned}
$$

where $\sigma_{i}$ are the spin variables. Equation (15) allows us to calculate the expectation value of the one-electron $z$ parity if we know the exact $N$-electron wave function. In general, the expectation value of $\hat{\pi}$ can take on arbitrary values within the interval $[-1,1]$. If the $N$-electron wave function is approximated by the Slater determinant $\Psi_{S}$, then the expectation value of the one-electron $z$ parity is equal to the arithmetic mean of the expectation values of the parities of $N$ one-electron wave functions, i.e.,

$$
\begin{aligned}
\langle\hat{\pi}\rangle & \equiv\left\langle\Psi_{S}|\hat{\pi}| \Psi_{S}\right\rangle \\
& =\frac{1}{N} \sum_{i=1}^{N} \int_{-\infty}^{+\infty} \int_{-\infty}^{+\infty} \int_{-\infty}^{+\infty} d x d y d z \psi_{i}^{\star}(x, y, z) \psi_{i}(x, y,-z),
\end{aligned}
$$

where $\psi_{i}(x, y, z)$ are the one-electron wave functions. If the electrons confined in the QDs occupy the even-parity states exclusively, the expectation value of the one-electron parity equals 1 . If however the electrons occupy both the even- and odd-parity states, the expectation value of the one-electron parity can be expressed as $\langle\hat{\pi}\rangle=\left(N_{\text {even }}-N_{\text {odd }}\right) / N$, where $N_{\text {even }}$ $\left(N_{\text {odd }}\right)$ is the number of electrons occupying the even (odd) parity states. We note that the one-electron $z$ parity $\hat{\pi}$ is closely related with the $z$ component of the isospin. ${ }^{31}$ The $z$-component isospin quantum number $I_{z}$ takes on the value $I_{z}=\left(N_{\text {even }}-N_{\text {odd }}\right) / 2 .{ }^{19}$

In the two vertically coupled QDs, there are only two regions, in which the electrons can be localized with a large probability. These regions are the lower QD, for which $z$ $<0$, and the upper QD, for which $z>0$. For the two electrons the probability to find exactly one electron in each QD is given by

$$
\begin{aligned}
P(1 \mid 1)= & \int_{z<0} d^{3} r \int_{z^{\prime}>0} d^{3} r^{\prime}\left|\Psi\left(\vec{r}, \vec{r}^{\prime}\right)\right|^{2} \\
& +\int_{z>0} d^{3} r \int_{z^{\prime}<0} d^{3} r^{\prime}\left|\Psi\left(\vec{r}, \vec{r}^{\prime}\right)\right|^{2},
\end{aligned}
$$

where $\Psi$ is the two-electron wave function. For the twoelectron system the probability of finding both electrons in the single QD is given by

$$
\begin{aligned}
P(0 \mid 2)= & \int_{z<0} d^{3} r \int_{z^{\prime}<0} d^{3} r^{\prime}\left|\Psi\left(\vec{r}, \vec{r}^{\prime}\right)\right|^{2} \\
& +\int_{z>0} d^{3} r \int_{z^{\prime}>0} d^{3} r^{\prime}\left|\Psi\left(\vec{r}, \vec{r}^{\prime}\right)\right|^{2} .
\end{aligned}
$$

Formulas (17) and (18) can be easily generalized to a system of $N>2$ electrons. Therefore, we obtain the relative probability $P\left(N_{1} \mid N_{2}\right)$ of finding $N_{1}$ electrons in the upper QD and $N_{2}$ electrons in the lower QD, where $N_{1}+N_{2}=N$. We note that-for each $N$-the sum of all probabilities $P\left(N_{1} \mid N_{2}\right)$ is equal to 1 .

\section{RESULTS}

\section{A. Two electrons}

The two-electron system confined in the coupled QDs is the simplest system, in which correlation plays an important role. The calculations for this system have been performed 
a)

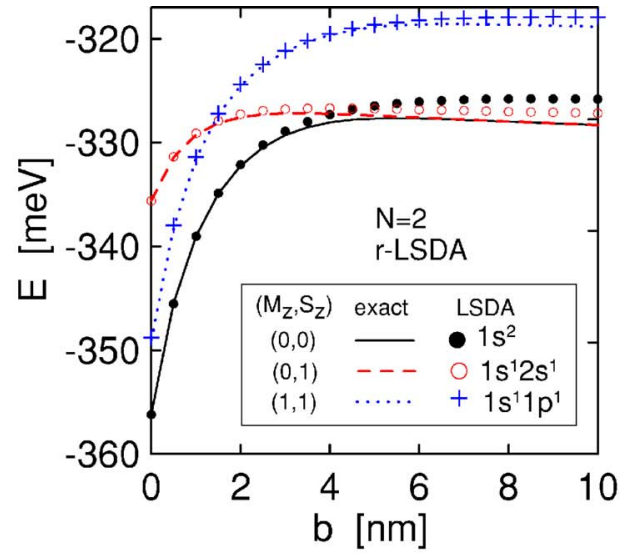

b)

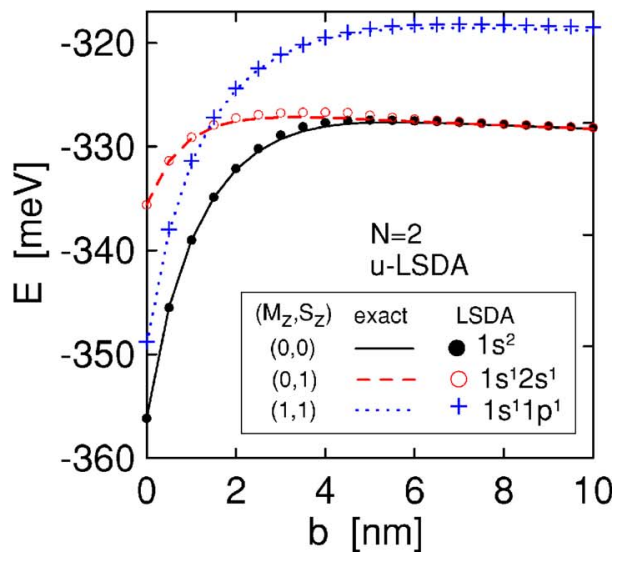

FIG. 1. (Color online) Lowest-energy levels of two electrons in coupled QDs calculated by the (a) $r$-LSDA and (b) $u$-LSDA as a function of interdot barrier thickness $b$. Exact results for states $\left(M_{z}, S_{z}\right)=(0,0),(0,1)$, and $(1,1)$ are plotted by solid, dashed, and dotted curves, respectively, the LSDA results for the corresponding states $1 s^{2}, 1 s^{1} 2 s^{1}$, and $1 s^{1} 1 p^{1}$ are shown by full dots, open dots, and crosses.

using the imaginary-time step method with the center-ofmass separation (called the "exact" method) and the two versions of the LSDA. Figures 1(a) and 1(b) show the barrierwidth dependence of the three lowest-energy levels obtained by these three methods. The ground state of the two-electron artificial molecule is the spin singlet with $M_{z}=0$ [cf. solid curves in Figs. 1(a) and 1(b)]. For wide barriers, i.e., for $b \geqslant 6 \mathrm{~nm}$, the two-electron molecule in the triplet state with $M_{z}=0$ has the same energy as in the $s$ singlet state, i.e., the ground state becomes fourfold degenerate. For narrow barriers the $r$-LSDA results are almost the same as the exact ones [Fig. 1(a)]. However, the errors of the $r$-LSDA energy estimates increase with increasing thickness of the barrier, which leads to qualitatively erroneous results, namely, the transition of the $s$-type ground-state configuration from a singlet to triplet state at $b=4.2 \mathrm{~nm}$. Figure 1(b) shows that the $u$-LSDA method correctly reproduces the energy-level sequence for all barrier thicknesses. For wide barriers the errors of the $u$-LSDA are several times smaller than the corresponding errors of the $r$-LSDA (Fig. 2). This means that-in contrast to the $r$-LSDA - the $u$-LSDA takes into account a major part of the electron-electron correlation. We note that-for the two lowest-energy levels-the $u$-LSDA energy errors pos-

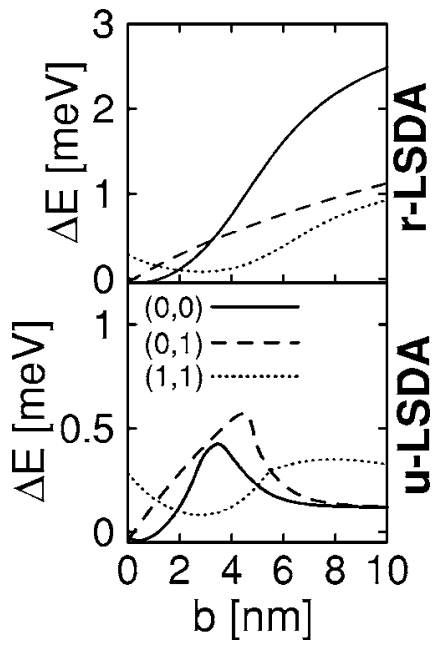

FIG. 2. Energy differences $\Delta E$ between the $r$-LSDA (upper panel) and $u$-LSDA (lower panel) and exact results for the lowestenergy levels of two electrons in coupled QDs as a function of interdot barrier thickness $b$. Solid, dashed, and dotted curves show the energy differences for states $(0,0),(0,1)$, and $(1,1)$, respectively.

sess a maximum at intermediate barrier thicknesses. It is interesting that the energy estimates obtained by the restricted and unrestricted Hartree-Fock methods, in the framework of the quasi-one-dimensional model of the two-electron coupled QDs, ${ }^{18}$ exhibit a similar qualitative behavior.

The correlation should also affect the symmetry of the wave function. In order to determine this effect we calculated the expectation value of the one-electron parity (16) [Fig. $3(\mathrm{a})$ ] and the relative probabilities (17) and (18) [Fig. 3(b)] by the exact and $u$-LSDA methods for the three lowestenergy states. For the state with $\left(M_{z}, S_{z}\right)=(0,0)$, i.e., $s$-type singlet, the expectation value of the one-electron parity [Fig. $3(\mathrm{a})$ ] is a monotonically decreasing function of the barrier thickness. It equals 1 for $b=0$ and tends to zero for wide barriers. The one-electron parity of state $(1,1)$, i.e., $p$-type triplet, exhibits similar properties, but it has larger values and more slowly tends to zero with increasing barrier width.

For state $(0,1)$, i.e., $s$-type triplet, the one-electron parity is always zero [cf. open dots in Fig. 3(a)]. We note that in this state the total isospin ${ }^{31}$ quantum number $I=0$ and is a good quantum number. In the $s$ triplet state, the $z$ component of the isospin $I_{z}$ also must be zero. Our results show that-in this two-electron state-both $I=0$ and $I_{z}=0$ do not change with varying barrier thickness.

Figure 3(b) shows that the relative probability $P(1 \mid 1)$ is always larger than $P(0 \mid 2)$, which means that electrons avoid each other and become localized in different QDs. For large barrier thickness this effect is stronger. For a sufficiently wide barrier $P(1 \mid 1)=1$ and $P(0 \mid 2)=0$, which implies that tunnel coupling vanishes and the two electrons become localized in separate dots. The properties of the relative probabilities of the $s$-type singlet and $p$-type triplet states are similar to each other; in particular, they are close to $1 / 2$ for $b=0$. In the $s$-type triplet state, the two-electron system exhibits a tendency towards electron separation already for $b=0$ [Fig. 3(b)].

The results obtained with the $u$-LSDA are considerably different from the exact results (Fig. 3). In the s-type singlet 
a)

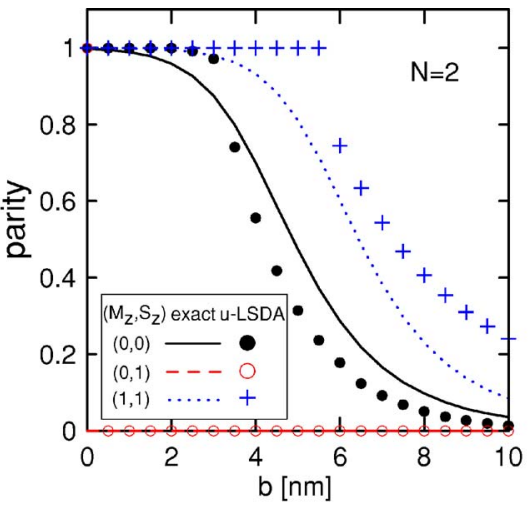

b)

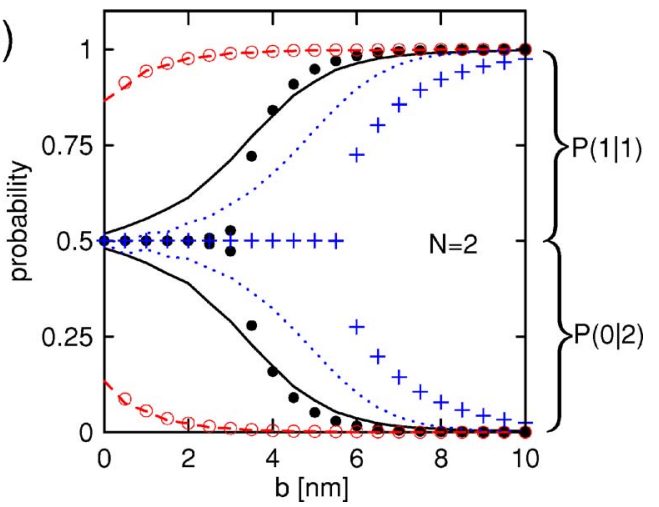

FIG. 3. (Color online) (a) Expectation value of the one-electron parity and (b) the relative probability of finding one electron in each QD, $P(1 \mid 1)$, and both electrons in one QD, $P(0 \mid 2)$, for the twoelectron system calculated by the exact method (solid, dashed, and dotted curves) and by the $u$-LSDA (dots and crosses) for the threelowest energy states, defined in the panel in part (a), as a function of interdot barrier thickness $b$. In part (a) the dotted curve for the state $(0,1)$ is not visible, since it coincides with the abscissa.

and $p$-type triplet states the expectation value of the oneelectron parity and the relative probability, calculated with the $u$-LSDA wave functions, stay constant for narrow barriers. These quantities begin to change for $b>2 \mathrm{~nm}$ in the $s$-type singlet state and for $b>6 \mathrm{~nm}$ in the $p$-type triplet state. Only for the $s$-type triplet states the $u$-LSDA approach correctly reproduces the exact results. If $P(1 \mid 1)=P(0 \mid 2)$ $=1 / 2$, the two-electron system can be treated as weakly correlated. Figure 3(b) shows that in the $u$-LSDA the twoelectron artificial molecule becomes weakly correlated for narrow barriers, since in this case $P(1 \mid 1) \simeq P(0 \mid 2) \simeq 1 / 2$. In the weakly correlated state, the one-electron wave functions possess definite parity [cf. Fig. 3(a)]. Deviation between the exact and $u$-LSDA results [Fig. 3(a)] indicates that the correlation breaks the one-electron parity already for intermediate interdot barrier thickness.

We conclude that the $u$-LSDA leads to much better estimates for the lowest-energy levels than the $r$-LSDA. However, the symmetry properties of the wave functions are not always correctly reproduced by the $u$-LSDA, in particular, for narrow interdot barriers.

\section{B. Three electrons}

In the three-electron system, the two lowest energy levels are associated with the configurations $M_{z}=0, S_{z}=1 / 2$ and a)

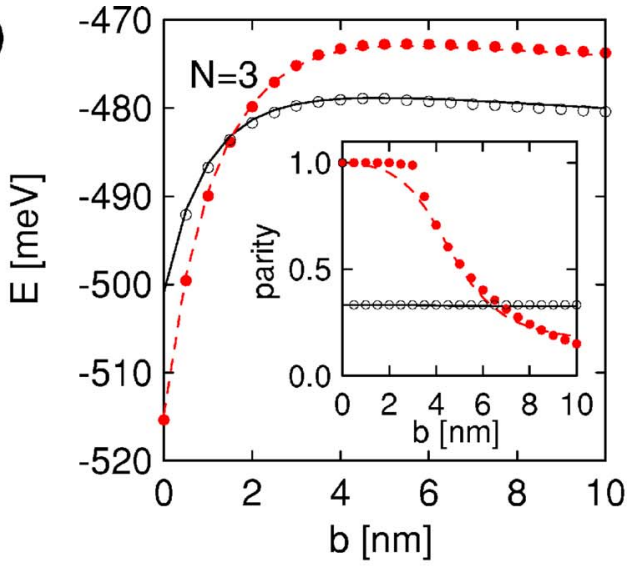

b)

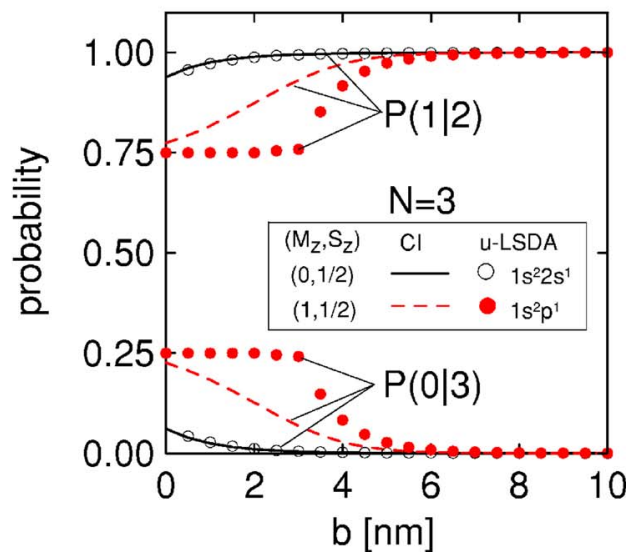

FIG. 4. (Color online) (a) Lowest-energy levels of three electrons in coupled QDs and (b) relative probabilities of finding one electron in one QD and two electrons in the second QD $[P(1 \mid 2)]$ and all three electrons in one $\mathrm{QD}[P(0 \mid 3)]$, for the lowest-energy states $(0,1 / 2)$ and $(1,1 / 2)$ calculated by the CI method (solid and dashed curves) and $u$-LSDA (dots) as a function of interdot barrier thickness $b$. Solid curve (open dots) corresponds to the state $(0,1 / 2)$ and dashed curve (full dots) corresponds to the state $(1,1 / 2)$. Inset in (a), expectation value of the one-electron parity calculated by the CI method (curves) and $u$-LSDA (dots) as a function of barrier thickness $b$.

$M_{z}=1, S_{z}=1 / 2$. Figure 4(a) displays these energy levels as a function of the barrier thickness calculated by the $u$-LSDA and CI methods. We see that independently of the barrier thickness the $u$-LSDA method accurately reproduces the energy levels and correctly predicts the ground-state transformation $(0,1 / 2) \rightarrow(1,1 / 2)$ at the same barrier thickness $(b$ $\simeq 1.5 \mathrm{~nm}$ ) as the CI method.

For the three-electron system we have also calculated the expectation value of the one-electron parity [inset in Fig. 4(a)] and the relative probability [Fig. 4(b)]. For state $(0,1 / 2)$, the one-electron parity is close to 1 for narrow interdot barriers and decreases with increasing barrier thickness $b$. For increasing $b$ the probability $P(0 \mid 3)$ of finding all three electrons in a single QD decreases, while the probability $P(1 \mid 2)$ of finding one electron in one QD and two electrons in the second QD increases. We obtain the same qualitative behavior of the relative probability for the state $(1,1 / 2)$. In this state the one-electron parity equals $1 / 3$ and 
a)

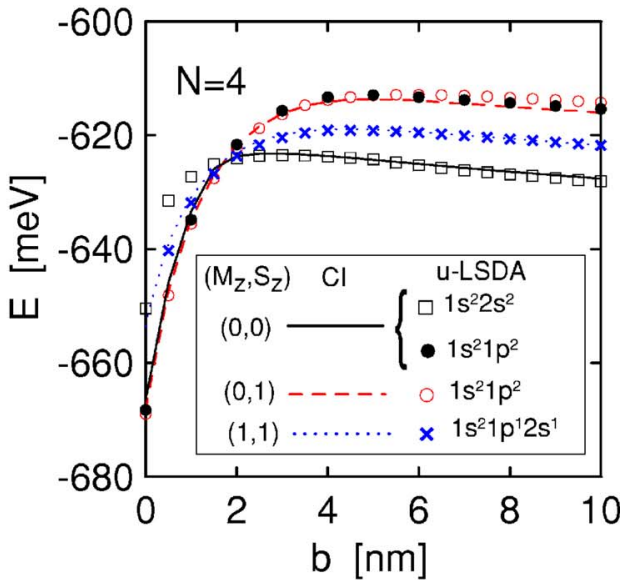

b)

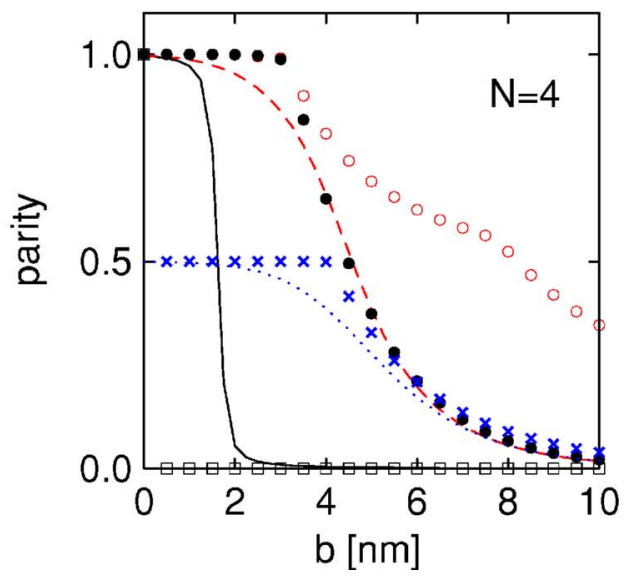

FIG. 5. (Color online) (a) Lowest-energy levels of four electrons in coupled QDs and (b) expectation value of one-electron parity as a function of the interdot barrier thickness $b$. Solid, dashed, and dotted curves display the CI results for $(0,0),(0,1)$, and $(1,1)$ states. Squares, full dots, open dots, and crosses show the $u$-LSDA results for configurations $1 s^{2} 2 s^{2}, 1 s^{2} 1 p^{2}$ (singlet), $1 s^{2} 1 p^{2}$ (triplet), and $1 s^{2} 1 p^{1} 2 s^{1}$.

does not change as a function of $b$ [cf. inset of Fig. 4(a)]. The one-electron parity calculated by the $u$-LSDA method differs from the exact result only in a narrow interval of intermediate barrier thickness. However, the relative probability is not well reproduced by the $u$-LSDA in the wider interval of barrier thickness [cf. Fig. 4(b)]. This means that the relative probability is more sensitive to the electron-electron correlation than the one-electron parity. Nevertheless, the $u$-LSDA method very well reproduces the one-electron parity and the relative probability in state $(0,1 / 2)$. Therefore, similarly as for the two-electron system, the $u$-LSDA method can reproduce the properties of the exact wave function in the entire range of interdot distance for the lowest-energy electronic configurations. For the higher-energy states, these properties are reproduced only for wide interdot barriers.

\section{Four electrons}

For the four electrons confined in the double coupled QD we consider the three following configurations: $\left(M_{z}, S_{z}\right)$ $=(0,0),(0,1)$, and $(1,1)$, which correspond to the lowest energy levels. The dependence of the energy levels on the bar- a)

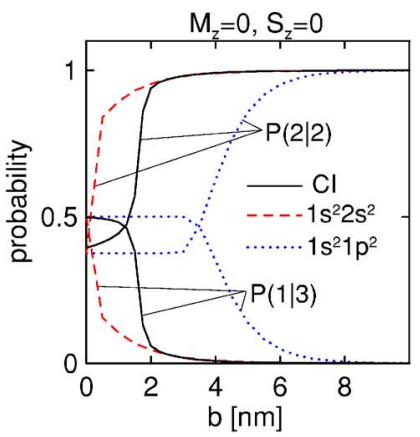

b)

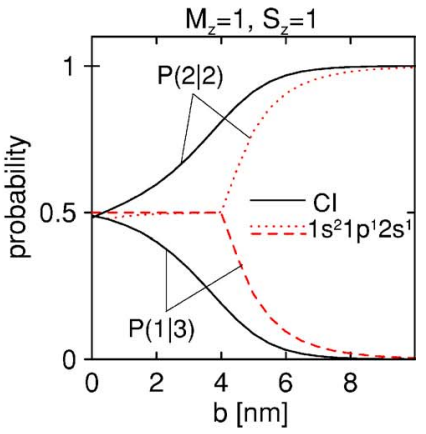

c)

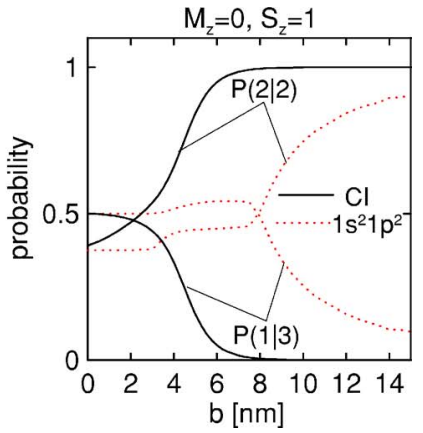

FIG. 6. (Color online) Relative probabilities of finding one electron in one QD and three electrons in the second QD $[P(1 \mid 3)]$ and the two electrons in each QD $[P(2 \mid 2)]$ calculated by the CI method (solid curves) and $u$-LSDA (dashed and dotted curves) as a function of the barrier thickness $b$ for four electrons with the quantum numbers (a) $M_{z}=0$ and $S_{z}=0$, (b) $M_{z}=1$ and $S_{z}=1$, and (c) $M_{z}=0$ and $S_{z}=1$.

rier thickness is plotted in Fig. 5(a). We observe that the $u$-LSDA method well reproduces the energy levels of states $(0,0)$ and $(1,1)$. Nevertheless, in the wide-barrier region, the $u$-LSDA energy estimate for the state $(0,1)$ is higher than the exact value. We also see that the lowest-energy spectrum is more complex than those for the two and three electrons. For example, in the $u$-LSDA calculations for the first singlet state with $M_{z}=0$, we must consider the two configurations: $1 s^{2} 1 p^{2}$ for $b \leqslant 1.7 \mathrm{~nm}$ and $1 s^{2} 2 s^{2}$ for wider barriers, which results from the fact that in the $u$-LSDA method the electron configuration of the given $\mathrm{N}$-electron state is fixed. On the contrary, within the CI method the electronic configuration is adjusted automatically by changing the coefficients in the linear combination of Slater determinants.

The results of the present calculations for the one-electron parity and relative probability are depicted in Figs. 5(b) and 6(a)-6(c). These figures show that the $u$-LSDA method reproduces the exact results for the ground state $(0,0)$ in the limit $b \rightarrow 0$ and for $b \geqslant 2 \mathrm{~nm}$. The corresponding configura- 


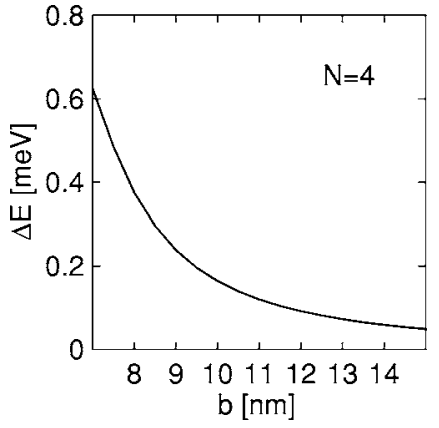

FIG. 7. Energy difference $\Delta E$ between the energy of two electrostatically coupled two-electron QDs $[P(2 \mid 2)=1]$ and the energy of four electrons in coupled QDs in a state with $M_{z}=0$ and $S_{z}=1$ as a function of barrier thickness $b$.

tions are $1 s^{2} 1 p^{2}$ in the former case and $1 s^{2} 2 s^{2}$ in the latter. Therefore, we can argue that -in the state $(0,0)$ - the dominating electron configuration is $1 s^{2} 1 p^{2}$ for very narrow barriers and $1 s^{2} 2 s^{2}$ for wide barriers. The $u$-LSDA method does not reproduce the one-electron parity and relative probability in state $(0,0)$ for $1 \mathrm{~nm} \leqslant b \leqslant 2 \mathrm{~nm}$. In this interval, both quantities change rapidly, but in a continuous manner, which is in contrast to the $u$-LSDA results that exhibit jumps.

We observe that-in the four-electron state $(0,0)$, which corresponds to the state $1 s^{2} 2 s^{2}$ in the $u$-LSDA-the oneelectron parity is zero. The same property has been found for the two-electron state $(0,1)$ (cf. Sec. III A). This means that $I=I_{z}=0$ and both isospin and its $z$ component are good quantum numbers.

Figure 5(b) shows that -in the state $(1,1)$ - the oneelectron parity obtained by the $u$-LSDA method considerably deviates from the exact values for $3 \mathrm{~nm} \leqslant b \leqslant 6 \mathrm{~nm}$. For other barrier thicknesses the deviations are small. Figure 6(b) shows that -in this state-relative probabilities $P(1 \mid 3)$ and $P(2 \mid 2)$ are well reproduced by the $u$-LSDA method if $b>8 \mathrm{~nm}$ and in the limit of zero barrier thickness. The largest deviations of the relative probabilities appear for intermediate barrier thickness, i.e., for $b \simeq 4 \mathrm{~nm}$, at which the fourelectron artificial molecule undergoes a transformation from the weakly correlated state (for small $b$ ) to the strongly correlated state (for large $b$ ).

Among the three configurations studied for the fourelectron system, the $u$-LSDA method cannot reproduce the one-electron parity and relative probability for the triplet state with $M_{z}=0$. Figures 5(b) and 6(c) show that only for $b<1 \mathrm{~nm}$ the one-electron parity and relative probability calculated by the $u$-LSDA approximate the exact results fairly well. If the barrier thickness increases, the deviations between the $u$-LSDA and exact results increase. This behavior is seen even for $b>3 \mathrm{~nm}$, for which the parity symmetry of the external potential is broken in the one-electron wave functions. In the interval $2 \mathrm{~nm} \leqslant b \leqslant 8 \mathrm{~nm}$, the $u$-LSDA relative probabilities slightly increase and $P(1 \mid 3)$ still remains larger than $P(2 \mid 2)$, contrary to the exact relative probabilities, which increase rapidly and $P(1 \mid 3)$ is considerably smaller than $P(2 \mid 2)$. The relative probabilities calculated by the $u$-LSDA are equal for $b \simeq 8 \mathrm{~nm}$ and only for $b \geqslant 8 \mathrm{~nm}$ $P(2 \mid 2)$ becomes larger than $P(1 \mid 3)$. For larger $b$ the devia- a)

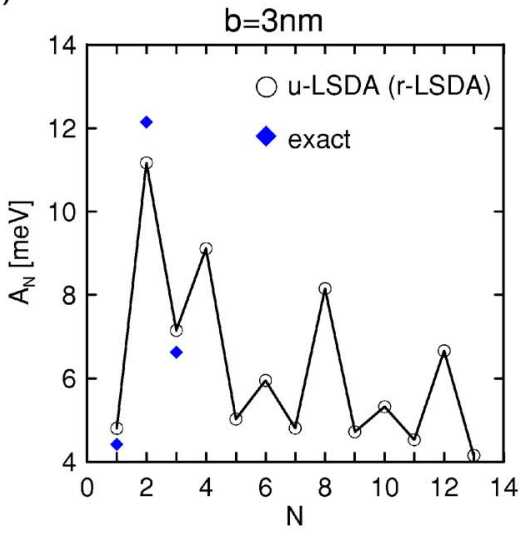

b)

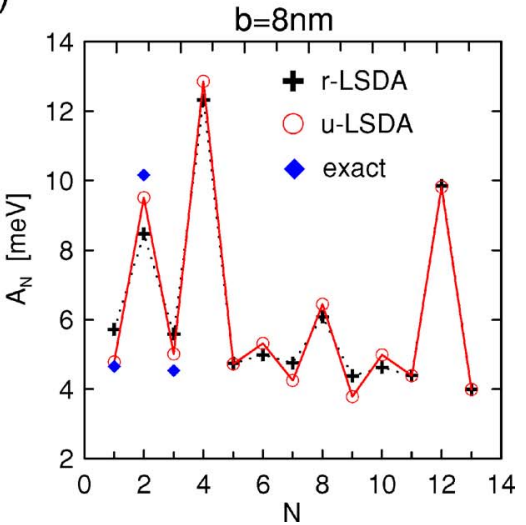

FIG. 8. (Color online) Addition energy $A_{N}$ calculated by the $u$-LSDA and $r$-LSDA for the $N$-electron coupled QDs separated by a barrier of thickness (a) $b=3 \mathrm{~nm}$ and (b) $b=8 \mathrm{~nm}$. For $N=1,2$, and 3 we also show the exact results. The lines are guides to the eye.

tions between the $u$-LSDA and the exact relative probabilities decrease, but still remain quite large.

We can explain this disagreement for the state $(0,1)$ by comparing the energy of this state calculated by the $u$-LSDA with the energy of the four-electron system, calculated under the requirement that the two electrons are localized in each QD. In the latter case, each of the two electrons occupies either the $1 s$ or $1 p$ state. The difference between the energies of these two configurations is depicted in Fig. 7. This energy difference decreases with increasing $b$ and becomes less than $0.4 \mathrm{meV}$ for wide barriers, i.e., for $b>8 \mathrm{~nm}$, for which the relative probability $P(2 \mid 2) \simeq 1$ [cf. Fig. 6(c)]. These results suggest that the $u$-LSDA method overestimates the exchange contribution, which causes the two electrons with the same spin to exhibit a tendency to be localized in the same QD. As a result, the $u$-LSDA approach cannot reproduce the oneelectron parity and the relative probabilities for the triplet state with $M_{z}=0$ even for wide interdot barriers.

\section{Addition energy for artificial molecules with $N=1, \ldots, 13$ electrons}

In transport spectroscopy experiments ${ }^{1,2,22}$ one can determine the change of the ground-state energy of the fewelectron system, when adding or removing a single electron. 
The relevant quantity is the addition energy $A_{N}$, i.e., the energy needed to add an extra electron to the $N$-electron QD. The addition energy is calculated as follows:

$$
A_{N}=E_{N+1}-2 E_{N}+E_{N-1},
$$

where $E_{N}$ is the ground-state energy of the $N$-electron system. The addition energy depends on the number of electrons confined in the QD system and the confinement potential profile. ${ }^{32}$ In the coupled QD nanostructure, the addition energy also depends on the thickness of the barrier separating the QDs. The dependence of the addition energy on the number $N$ of electrons confined in the QDs is characteristic for the QD system considered.

We calculated the addition energy for a system of $N=1, \ldots, 13$ electrons confined in two vertically coupled QDs using both the $u$-LSDA and $r$-LSDA methods. The results are depicted in Fig. 8, which also shows the exact results for $A_{1}, A_{2}$, and $A_{3}$ calculated using the methods described in the preceding sections. When studying the twoelectron coupled QDs, we found that for narrow barriers both LSDA methods provide good approximations to the exact results. Figure 8(a) supports this conclusion: for narrow interdot barrier both the LSDA methods lead to identical addition energies, which only slightly differ from the exact results for $N=1,2$, and 3. For large interdot barrier [Fig. 8(b)] there appear small differences between the addition energies calculated by both the LSDA methods and the $u$-LSDA estimates are closer to the exact values. However, the differences do not exceed $1 \mathrm{meV}$ and do not change the qualitative dependence of the addition energy on $N$.

For wide barriers we found that-according to the $r$-LSDA and $u$-LSDA methods-subsequent orbitals become occupied in a different manner. In the $u$-LSDA the electrons occupy subsequent orbitals, i.e., in the following order: $1 s, 2 s, 1 p, 2 p, 1 d, \ldots$. This is different from the $r$-LSDA approach where the next orbital can be occupied before the former is fully filled. For example, for $N=2$ we obtain the lowest-energy configuration $1 s 2 s$, for which the $z$ component of the total spin is increasing $\left(S_{z}=1\right)$. Also for $N=7$ and $N$ $=8$, according to the $r$-LSDA, the electrons occupy the $2 p$ orbital, although the $1 p$ orbital is only half-filled. In this way, the $z$ component of the total spin increases: $S_{z}=3 / 2$ for $N$ $=7$ and $S_{z}=2$ for $N=8$. In the $r$-LSDA the empty $1 p$ states become occupied for $N=9$ and $N=10$. We conclude that in the $r$-LSDA the electrons exhibit a too large preference to occupy states with the same spin, which leads to an enhancement of the exchange interaction and a lowering of the ground state energy. Figure 8(b) shows that-in coupled QDs with wide interdot barrier-the addition energies calculated by the $r$-LSDA and $u$-LSDA methods are the same for $N=10,11$, and 12 .

The general features of the addition energy as a function of $N$ are the same for the narrow- and wide-barrier QD nanostructure. In particular, the addition energy is considerably larger for the closed-shell systems, i.e., for $N=2,4,8$, and 12 and slightly larger for the half-filled shells, i.e., for $N=6$ and 10. This behaviour of the coupled QD system is similar to that of the single $\mathrm{QD}^{32}$ and qualitatively agrees with the experimental data. ${ }^{1}$

\section{CONCLUSIONS}

In the present paper, we have studied few-electron systems confined in vertically coupled QDs using the exact and LSDA methods. We have applied two versions of the LSDA: the restricted LSDA, in which the one-electron wave functions reproduce the symmetry of the confinement potential, and the unrestricted LSDA, in which this symmetry is broken. A comparison with the exact results has allowed us to test the $r$-LSDA and $u$-LSDA methods. We have checked to which extend the LSDA wave functions reproduce the oneelectron properties of the exact $N$-electron wave function for the lowest-energy configurations of the coupled QD nanostructures with $N=2,3$, and 4 electrons. For this purpose we have calculated the one-electron parity and the relative probability of finding $N_{1}$ electrons in one QD and $N_{2}$ electrons in the other. It appears that the $u$-LSDA method very well reproduces both these quantities for the two-electron configuration with $M_{z}=0$ and $S_{z}=1$ and for the three-electron configuration with $M_{z}=0$ and $S_{z}=1 / 2$ for arbitrary interdotbarrier thickness, and for the four-electron configuration $M_{z}=0$ and $S_{z}=1$ when $b>2 \mathrm{~nm}$. Nevertheless, for the other configurations the one-electron parity and the relative probability considerably deviates from the exact values in the regime of small and intermediate barrier thicknesses, for which tunnel coupling between the QDs is strong. The exact results are reproduced by the $u$-LSDA at large barrier thicknesses, for which the QDs are only electrostatically coupled.

We have found that - for certain states of the two- and four-electron coupled QDs-the one-electron parity is equal to zero and does not change with the barrier thickness. We can interpret this effect in terms of isospin, which is also zero and which turns out to be a good quantum number in these quantum states.

The energies of the two-, three-, and four-electron systems in two coupled QDs calculated by the LSDA methods have been compared with the exact values. This comparison shows that the $u$-LSDA method quite well reproduces the lowest-energy levels. In the regime of narrow barriers, in which the one-electron wave functions possess a welldefined parity symmetry, the $u$-LSDA and $r$-LSDA methods are equivalent. For wide barriers the symmetry of the oneelectron wave functions is lowered, which is the reason why the $u$-LSDA method is much more accurate than the $r$-LSDA. This implies that the $u$-LSDA method much better takes into account correlation effects.

In summary, we have shown that-although the lowestenergy levels of the few-electron systems confined in coupled QDs are correctly reproduced by the unrestricted LSDA method-the one-electron parity and the relative probability calculated by the LSDA can considerably deviate from the exact values for some states and for intermediate values of the barrier thickness. The one-electron parity and the relative probability, introduced in the present paper, allow us to determine to which extent the symmetry properties of the exact many-electron solutions are recovered by the mean-field one-electron LSDA method. We conclude that the LSDA method should be used with some caution for coupled QDs. In particular, the one-electron symmetry properties are not always properly reproduced by the LSDA method. 


\section{ACKNOWLEDGMENTS}

The paper has been partly supported by the Polish Government Scientific Committee Grant No. KBN 1P03B06126, the Flemish Science Foundation (FWO-Vl), and the Belgian Science Policy. One of the authors (B.S.) is supported by the EC Marie Curie IEF project MEIF-CT-2004-500157.

\section{APPENDIX}

In the CI calculations, we need the matrix elements of the electron-electron Coulomb interaction

$$
\begin{aligned}
C_{a b c d}= & \int d^{6} r f_{a}\left(z_{1}\right) g_{a}^{*}\left(x_{1}, y_{1}\right) f_{b}\left(z_{2}\right) g_{b}^{*}\left(x_{2}, y_{2}\right) \\
& \times \frac{1}{r_{12}} f_{c}\left(z_{1}\right) g_{c}\left(x_{1}, y_{1}\right) f_{d}\left(z_{2}\right) g_{d}\left(x_{2}, y_{2}\right) .
\end{aligned}
$$

These matrix elements can be evaluated with the help of the effective interaction potential $V_{\text {eff }}^{a b c d}\left(\left|z_{1}-z_{2}\right|\right)$, which is derived using integration over the lateral degrees of freedom, ${ }^{33}$

$$
C_{a b c d}=\int d z_{1} d z_{2} f_{a}\left(z_{1}\right) f_{b}\left(z_{2}\right) V_{\mathrm{eff}}^{a b c d}\left(\left|z_{1}-z_{2}\right|\right) f_{c}\left(z_{1}\right) f_{d}\left(z_{2}\right)
$$

The integration over $z_{1}$ and $z_{2}$ is performed numerically. The explicit forms of the effective interaction potentials are (cf. Ref. 33)

$$
\begin{aligned}
& V_{\mathrm{eff}}^{0000}(z)=\sqrt{\pi \beta} \operatorname{erfcx}\left(\sqrt{\beta z^{2}}\right) \\
& V_{\mathrm{eff}}^{1010}(z)= \sqrt{\pi \beta}\left(3 / 4-\beta z^{2} / 2\right) \operatorname{erfcx}\left(\sqrt{\beta z^{2}}\right)+\beta z / 2, \\
& V_{\mathrm{eff}}^{1001}(z)= \sqrt{\pi \beta}\left(1 / 4+\beta z^{2} / 2\right) \operatorname{erfcx}\left(\sqrt{\beta z^{2}}\right)-\beta z / 2, \\
& V_{\mathrm{eff}}^{1,-1,1,-1}(z)= \sqrt{\pi \beta}\left(11 / 16-\beta z^{2} / 4+\beta^{2} z^{4} / 4\right) \operatorname{erfcx}\left(\sqrt{\beta z^{2}}\right) \\
&+3 \beta z / 8-\beta^{2} z^{3} / 4, \\
& V_{\mathrm{eff}}^{1,-1,-1,1}(z)= \sqrt{\pi \beta}\left(3 / 16+3 \beta z^{2} / 4+\beta^{2} z^{4} / 4\right) \operatorname{erfcx}\left(\sqrt{\beta z^{2}}\right) \\
&-5 \beta z / 8-\beta^{2} z^{3} / 4
\end{aligned}
$$

*Electronic address: adamowski@ftj.agh.edu.pl

†Electronic address: francois.peeters@ua.ac.be

${ }^{1}$ D. G. Austing, T. Honda, K. Muraki, Y. Tokura, and S. Tarucha, Physica B 249-251, 206 (1998)

${ }^{2}$ S. Tarucha, T. Honda, D. G. Austing, Y. Tokura, K. Muraki, T. H. Oosterkamp, J. W. Janssen, and L. P. Kouwenhoven, Photonics Spectra 112, 120 (1998).

${ }^{3}$ L.-X. Zhang, P. Matagne, J. P. Leburton, R. Hanson, and L. P. Kouwenhoven, Phys. Rev. B 69, 245301 (2004).

${ }^{4}$ A. W. Rushforth, C. G. Smith, M. D. Godfrey, H. E. Beere, D. A. Ritchie, and M. Pepper, Phys. Rev. B 69, 113309 (2004).

${ }^{5}$ M.-R. Li and K. Le Hur, Phys. Rev. Lett. 93, 176802 (2004).

${ }^{6}$ G. Ortner, R. Oulton, H. Kurtze, M. Schwab, D. R. Yakovlev, M. Bayer, S. Fafard, Z. Wasilewski, and P. Hawrylak, Phys. Rev. B 72, 165353 (2005).

${ }^{7}$ B. D. Geradot, S. Strauf, M. J. A. de Dood, A. M. Bychkov, A. Badolato, K. Hennessy, E. L. Hu, D. Bouwmeester, and P. M. Petroff, Phys. Rev. Lett. 95, 137403 (2005).

${ }^{8}$ J. Fabian and U. Hohenester, Phys. Rev. B 72, 201304(R) (2005).

${ }^{9}$ R. Requist, J. Schliemann, A. G. Abanov, and D. Loss, Phys. Rev. B 71, 115315 (2005).

${ }^{10}$ H.-M. Müller and S. E. Koonin, Phys. Rev. B 54, 14532 (1996).

${ }^{11}$ C. Yannouleas and U. Landman, Phys. Rev. Lett. 82, 5325 (1999)

${ }^{12}$ C. Yannouleas and U. Landman, Eur. Phys. J. D 16, 373 (2001).

${ }^{13}$ M. Pi, A. Emperador, M. Barranco, and F. Garcias, Phys. Rev. B 63, 115316 (2001).

${ }^{14}$ M. Pi, A. Emperador, M. Barranco, F. Garcias, K. Muraki, S. Tarucha, and D. G. Austing, Phys. Rev. Lett. 87, 066801 (2001).

${ }^{15}$ B. Partoens and F. M. Peeters, Phys. Rev. Lett. 84, 4433 (2000).

${ }^{16}$ A. Wensauer, O. Steffens, M. Suhrke, and U. Rössler, Phys. Rev. B 62, 2605 (2000).
${ }^{17}$ A. Harju, E. Räsänen, H. Saarikoski, M. J. Puska, R. M. Nieminen, and K. Niemelä, Phys. Rev. B 69, 153101 (2004).

${ }^{18}$ S. Bednarek, T. Chwiej, J. Adamowski, and B. Szafran, Phys. Rev. B 67, 205316 (2003).

${ }^{19}$ M. Rontani, S. Amaha, K. Muraki, F. Manghi, E. Molinari, S. Tarucha, and D. G. Austing, Phys. Rev. B 69, 085327 (2004).

${ }^{20}$ M. Helle, A. Haraju, and R. M. Nieminen, Phys. Rev. B 72, 205329 (2005).

${ }^{21}$ B. Partoens and F. M. Peeters, Physica B 298, 282 (2001); B. Partoens and F. M. Peeters, Europhys. Lett. 56, 86 (2001).

${ }^{22}$ D. G. Austing, S. Tarucha, H. Tamura, K. Muraki, F. Ancilotto, M. Barranco, A. Emperador, R. Mayol, and M. Pi, Phys. Rev. B 70, 045324 (2004).

${ }^{23}$ H. Tamura, Physica B 248-251, 210 (1998).

${ }^{24}$ B. Partoens, A. Matulis, and F. M. Peeters, Phys. Rev. B 59, 1617 (1999).

${ }^{25}$ H. Saarikoski, E. Räsänen, S. Siljamaki, A. Harju, M. J. Puska, and R. M. Nieminen, Eur. Phys. J. B 26, 241 (2002).

${ }^{26}$ E. Räsänen, H. Saarikoski, V. N. Stavrou, A. Harju, M. J. Puska, and R. M. Nieminen, Phys. Rev. B 67, 235307 (2003).

${ }^{27}$ B. Szafran, J. Adamowski, and S. Bednarek, Physica E (Amsterdam) 5, 185 (2000).

${ }^{28}$ K. T. Davies, H. Flocard, S. Krieger, and M. S. Weiss, Nucl. Phys. A 342, 112 (1980).

${ }^{29}$ J. P. Perdew and A. Zunger, Phys. Rev. B 23, 5048 (1981).

${ }^{30}$ D. M. Ceperley and B. J. Alder, Phys. Rev. Lett. 45, 566 (1980).

${ }^{31}$ J. J. Palacios and P. Hawrylak, Phys. Rev. B 51, 1769 (1995).

${ }^{32}$ S. Bednarek, B. Szafran, and J. Adamowski, Phys. Rev. B 64, 195303 (2001).

${ }^{33}$ S. Bednarek, B. Szafran, T. Chwiej, and J. Adamowski, Phys. Rev. B 68, 045328 (2003). 